\title{
A potential biomarker based on clinical-radiomics nomogram for predicting survival and adjuvant chemotherapy benefit in resected node-negative, early-stage lung adenocarcinoma
}

\author{
Xiaoling Ma ${ }^{1}$, Wenzhi $\mathrm{Lv}^{2}$, Cong Wang ${ }^{3}$, Dehao Tu${ }^{4}$, Jinhan Qiao ${ }^{1}$, Chanyuan Fan ${ }^{1}$ Jiandong Niu ${ }^{5}$, \\ Wen Zhou ${ }^{1}$, Qiuyu Liu ${ }^{1}$, Liming Xia ${ }^{1}$ \\ ${ }^{1}$ Department of Radiology, Tongji Hospital, Tongji Medical College, Huazhong University of Science and Technology, Wuhan, China; ${ }^{2}$ Department \\ of Artificial Intelligence, Julei Technology Company, Wuhan, China; ${ }^{3}$ Jiangsu Key Laboratory of Medical Optics, Suzhou Institute of Biomedical \\ Engineering and Technology, Chinese Academy of Sciences, Suzhou, China; ${ }^{4}$ Department of Thoracic surgery, Tongji Hospital, Tongji Medical \\ College, Huazhong University of Science and Technology, Wuhan, China; ${ }^{5}$ Department of Radiology, The General Hospital of Ningxia Medical \\ University, Yinchuan, China \\ Contributions: (I) Conception and design: X Ma; (II) Administrative support: L Xia; (III) Provision of study materials or patients: X Ma, W Lv, \\ C Wang; (IV) Collection and assembly of data: X Ma, J Qiao, C Fan, W Zhou, Q Liu; (V) Data analysis and interpretation: X Ma, W Lv, J Niu, \\ L Xia; (VI) Manuscript writing: All authors; (VII) Final approval of manuscript: All authors. \\ Correspondence to: Liming Xia. Department of Radiology, Tongji Hospital, Tongji Medical College, Huazhong University of Science and Technology, \\ 1095 Jiefang Road, Qiaokou District, Wuhan 430030, China. Email: xialiming2017@outlook.com.
}

Background: We aimed to construct a clinical-radiomics nomogram to predict disease-free survival (DFS) and the added survival benefit of adjuvant chemotherapy (ACT) for node-negative, early-stage (I-II) lung adenocarcinoma (ADC).

Methods: In this retrospective study including 310 patients from two independent cohorts, the CTderived radiomics features were selected by least absolute shrinkage and selection operator Cox regression to generate a radiomics signature associated with DFS. The radiomics signature was incorporated to construct a clinical-radiomics nomogram along with the independent clinical risk predictors. The model performance was evaluated with reference to discrimination quantified by Harrell concordance index (C-index), integrated discrimination improvement (IDI) and net reclassification index (NRI), calibration and clinical utility. The risk score (RS) for clinical-radiomics nomogram was calculated. The association between ACT and survival benefit was assessed in high and low RS subgroup.

Results: The clinical-radiomics nomogram achieved the highest C-index of 0.822 [95\% confidence interval (CI): $0.769,0.876$ ] in training cohort and 0.802 (95\% CI: $0.716,0.888)$ in validation cohort. The incorporation of radiomics signature into clinical-radiomics nomogram showed an incremental benefit over clinical nomogram according to the improved NRI and IDI. The calibration curves and decision curve analysis further verified the clinical utility of clinical-radiomics nomogram. Further, patients with high RS based on clinical-radiomics nomogram were more prone to benefit from ACT.

Conclusions: The clinical-radiomics nomogram approach can feasibly conduct risk prediction and have potential to identify the beneficiaries of ACT among patients with node-negative, early-stage ADC, which might serve as a helpful tool in informing therapeutic decision-making.

Keywords: Adjuvant chemotherapy (ACT); radiomics; survival; lung adenocarcinoma (lung ADC); computer tomography

Submitted Sep 19, 2021. Accepted for publication Dec 15, 2021.

doi: $10.21037 /$ jtd-21-1520

View this article at: https://dx.doi.org/10.21037/jtd-21-1520

(c) Journal of Thoracic Disease. All rights reserved. 


\section{Introduction}

Lung cancer remains the leading lethal malignancy, responsible for $18.4 \%$ of all cancer-related deaths worldwide in 2018 (1). Non-small cell lung cancer (NSCLC) is in the majority of all lung cancer cases, accounting for approximately $85 \%$ (2). Surgical resection is the prime therapeutic modality for early-stage (I-II) NSCLC (3). Despite generally prescribed with curing excision, earlystage NSCLC remains at a substantial risk of recurrence. Even in stage I NSCLC, more than $30 \%$ of patients will suffer a relapse after surgery within 5 years (4).

The tumor-node-metastasis (TNM) classification is regarded as the dominant reference criteria for prognosis evaluation in NSCLC. Given the heterogeneous outcomes of NSCLC patients even in identical pathological stage, it is insufficient to estimate prognosis and direct treatment merely depending on TNM classification system. Adjuvant chemotherapy (ACT) is an additional therapeutic option for lung cancer. Several large-sample, randomized controlled, clinical trials have reported the survival benefit of platinumbased ACT following surgery in patients with NSCLC (5-8). ACT is generally recommended for resected stage II-III patients (9), but there is still an unsolved debate on the indication of ACT in node-negative, early-stage NSCLC, especially in stage I cases. A comprehensive analysis based on data from The National Cancer Database, observed a survival benefit associated with ACT in patients with tumors $3 \mathrm{~cm}$ or larger and high-risk clinicopathological factors (10). Krivitsky et al. constructed a risk stratification model for negative-node NSCLC and assigned $9 \%$ of stage I patients at high-risk who could be potential candidates for ACT (11). More interestingly, series studies demonstrated the improved survival associated with ACT even in stage IA patients with high-risk histopathological factors $(12,13)$.

These discrepant propositions indicate the compelling necessity to refine risk stratification, intended to recognize patients at a substantial risk of recurrence as target candidates for more strict management and individualized therapy. Several efforts have been put into developing prognostic biomarkers, including genetic phenotypes $(4,14)$, cytokines (15) and serological indicators (16), but none of these established biomarkers can be clinically practical prior to laboratory testing standardization and trial validation (17). "Radiomics" is an emerging translational field of research widely applied in various aspects of medicine domain. Quantitative radiomics features excavated from multimodality medical images can noninvasively embody abundant information underlying pathophysiology and habitat microenvironment within tumors, which are hard to be appreciated by human naked eyes $(18,19)$. Lung adenocarcinoma (ADC) accounting for approximately $60 \%$ of NSCLC has distinct characteristics in histological architecture and molecular expression from other subtypes of lung cancer (20). This study sought to develop and validate a prognostic model based on the radiomics signature and clinical risk predictors to predict survival and conduct risk stratification in node-negative, early-stage ADC. It is hypothesized that this prognostic model could effectively identify potential beneficiaries of ACT among node-negative, early-stage ADC patients.

We present the following article in accordance with the TRIPOD reporting checklist (available at https://jtd. amegroups.com/article/view/10.21037/jtd-21-1520/rc).

\section{Methods}

\section{Patients}

The study was conducted in accordance with the Declaration of Helsinki (as revised in 2013). The Institutional Ethics Committee of Tongji Hospital approved this retrospective study (No. TJ-IRB-20180615) and the requirement for informed consent was waived. We retrospectively reviewed 4,124 patients receiving tumor radical resection and systematic lymph nodes dissection in Tongji hospital affiliated to Huazhong University of Science and Technology from May 2012 to September 2016. Totally, 186 eligible patients from 2,561 patients undergoing preoperative contrast-enhanced CT examination on the multi-slice spiral CT scanner of GE Discovery CT 750 HD (CT scanner 1) were included and constituted the training cohort. We enrolled 124 eligible patients from 1,563 patients receiving preoperative contrast-enhanced CT examination on the multi-slice spiral CT scanner of TOSHIBA Aquilion One (CT scanner 2) to set up the validation cohort. The inclusion criteria consisted of: (I) pathologically confirmed early-stage (III) ADC with negative margin and without regional lymph node and distant metastasis (N0M0R0); (II) no presurgical radiotherapy/chemotherapy and postsurgical radiotherapy/ targeted therapy; (III) within interval time of 2 weeks between presurgical contrast-enhanced CT examination and surgical resection. Tumor stage was determined according to the eighth edition of TNM classification for lung cancer. We excluded the patients: (I) with ADC in situ, minimally invasive ADC, the rare variants of ADC including mucinous, cribriform, colloidal, fetal and 


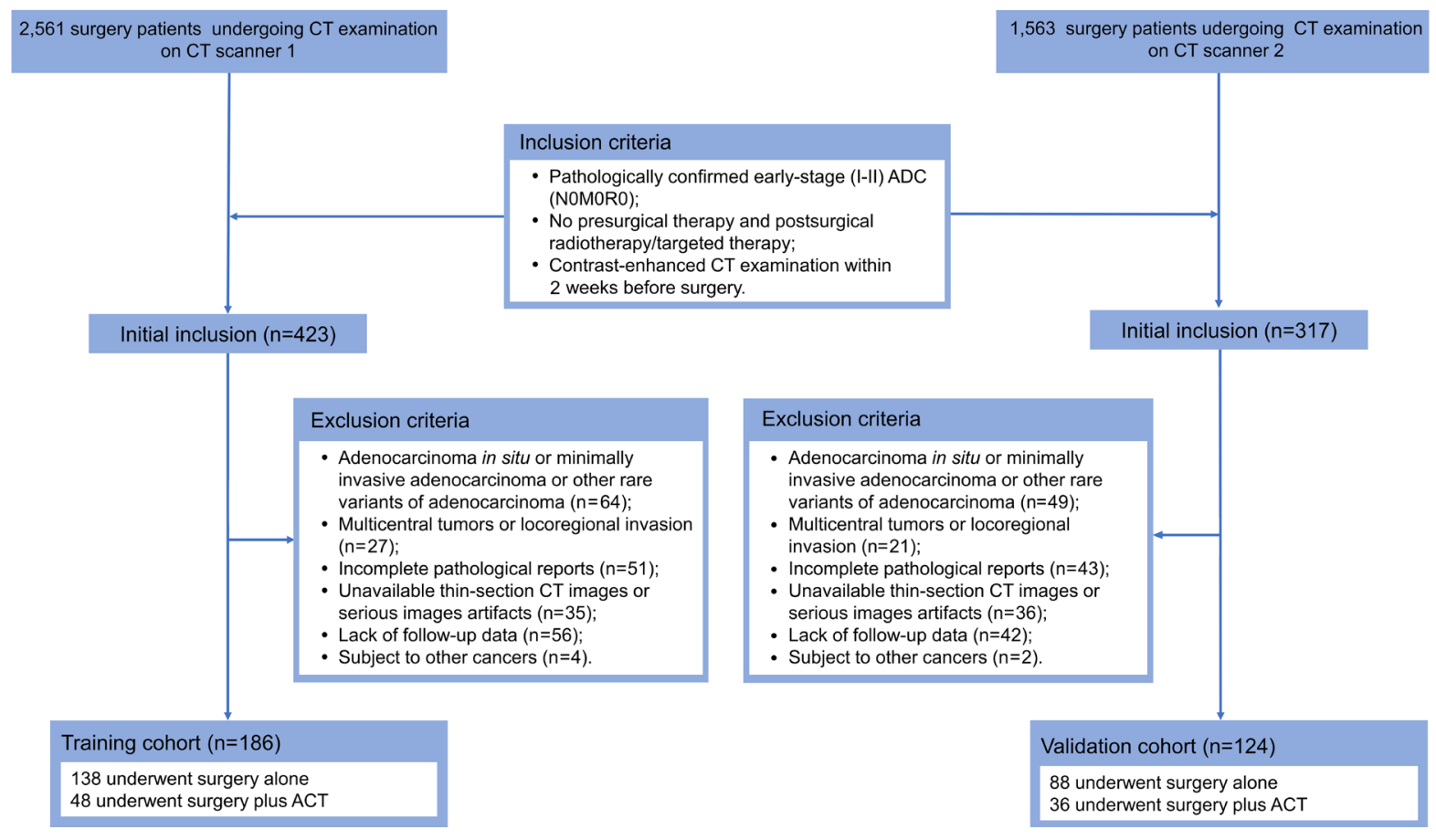

Figure 1 The recruitment pathway of this study. CT, computer tomography; ADC, adenocarcinoma; ACT, adjuvant chemotherapy.

intestinal ADC; (II) with two or more metachronous and synchronous primary tumors or locoregional invasion; (III) incomplete pathological reports; (IV) presurgical thinsection CT images were unavailable or CT images were highly distorted by motion artifacts; (V) lack of follow-up data; (VI) subjected to other cancers.

Figure 1 showed the recruitment pathway. The baseline characteristics including gender, age, smoking history, pack-year, histological grade, preoperative serum carcinoembryonic antigen (CEA) status, Ki-67 labeling index (LI), tumor size, pathological TNM stage, epidermal growth factor receptor (EGFR) mutation status, follow-up time and therapy were recorded. Histological grade is the prognostic classification of $\mathrm{ADC}$ predominant growth patterns, which separates invasive ADC into three prognostic grades: low grade (lepidic predominant ADC), intermediate grade (acinar or papillary predominant $\mathrm{ADC}$ ) and high grade (solid or micropapillary predominant ADC) (21).

\section{Follow-up}

Patients were followed up from the date of surgery every 6 months within the first 2 years, and then every 12 months. The follow-up time was shortest for 36 months, longest for 87 months. The follow-up evaluation included physical condition, thoracic CT examination (brain CT scan and abdominal ultrasound if required), serum tumor markers and postsurgical therapy. The endpoint, disease-free survival (DFS) was defined as the time from the date of surgery to the date of event or being censored. Tumor locoregional relapse, distant metastasis or death was regarded as the endpoint event. Patients last known to be alive free from recurrence were censored.

\section{Imaging acquisition and radiomics analysis}

All subjects underwent preoperative contrast-enhanced CT examination by the multi-slice spiral CT scanners of GE Discovery CT $750 \mathrm{HD}$ and TOSHIBA Aquilion One. The scanning parameters across different scanners or vendors always keep consistent as revealed in Appendix 1.

An open-source software, ITK-SNAP (version 3.8.0) was utilized to perform images segmentation. Two thoracic radiologists (M.X.L. and Z.W., with 8 and 3 years of experience) manually delineated the boundaries of tumors as region of interests on axial CT images in lung window (width, 1,500 HU; level, -500 HU) slice-byslice independently without the knowledge of the baseline characteristics. Each region of interest was drawn close to but not beyond the boundaries of tumors as far as possible, 
avoiding bronchus, vessels and necrosis in tumors. The radiomics feature extraction from the arterial phase $(30 \mathrm{~s}$ after contrast agent injection) images was conducted with a free open-source package of PyRadiomic (available at https://pyradiomics.readthedocs.io/en/latest/index.html) implemented in platform of Python (version 3.6.2), which is a popular programming language for scientific computation based on robust ecosystem and could be available on any system (22).

\section{Features selection and radiomics signature development}

The interclass correlation coefficients (ICCs) were respectively calculated to assess the interobserver agreement. To ensure the stability of features, radiomics features with ICC $<0.8$ were eliminated from the radiomics dataset. Then we calculated the Spearman correlation coefficient of paired features and excluded the redundant features with Spearman correlation coefficient $>0.7$. We conducted data normalization with the approach of $\mathrm{Z}$-score to achieve a standard distribution of feature value, the rationale of which is transforming different magnitude data into unified measurement for comparison. The least absolute shrinkage and selection operator (LASSO) Cox regression algorithm with 10 -fold cross validation was utilized to select the radiomics features with non-zero coefficients. Then we developed a radiomics signature denoted as radiomics score (Rad-score) by a linear combination of the selected features weighted by corresponding LASSO coefficients. The Rad-score was binarized at cut-off point to stratify patients into high Rad-score and low Rad-score subgroups in training cohort. The $\mathrm{X}$-tile software (version 3.6.1, Yale University, USA) was used to determine the cut-off point, where survival between two divided subgroups were best separated with the maximum chi-square value in log-rank test. Subsequently, the survival difference between high Rad-score and low Rad-score subgroup was assessed in validation cohort using Kaplan-Meier curves with log-rank tests. The prognostic performance of radiomics signature was analyzed in both training cohort and validation cohort.

\section{The clinical nomogram and clinical-radiomics nomogram construction}

Univariate and multivariate Cox regression analysis were used to identify independent clinical risk predictors associated with DFS. Then the clinical risk predictors were incorporated to construct a clinical nomogram based on multivariate Cox proportional hazards analysis with regression coefficients. Similarly, a clinical-radiomics nomogram was constructed integrating clinical risk predictors along with radiomics signature.

The Harrell concordance index (C-index) was calculated to quantify the discriminative ability of prediction models. To quantify the incremental value of radiomics signature in predictive accuracy, we also calculated the integrated discrimination improvement (IDI) and net reclassification index (NRI). The two assessment indexes, as an effective supplement to area under the receiver operating characteristic curve/C-index, were used to estimate performance improvement for models when introducing a new variable (23). The calibration curves for nomograms were depicted to assess the agreement between predicted probabilities with nomograms and actual observed probabilities. The decision curve analysis was conducted to exhibit the clinical utility of prediction models by assessing the overall benefits across a range of reasonable diagnostic thresholds in comprehensive consideration of insufficient treatment (false negative) and excessive treatment (false positive) (24).

\section{Clinical utility of nomogram in estimating survival benefit of ACT}

To estimate survival benefit of ACT, risk score (RS) for clinical-radiomics nomogram was calculated with a linear polynomial of corresponding risk predictors weighted by respective Cox regression coefficients. Patients were categorized into high RS and low RS subgroup by the optimal threshold determined by the X-tile. Then DFS of all patients between patients receiving surgery with ACT and without ACT in low RS or high RS subgroup was analyzed using Kaplan-Meier curves and Cox proportional hazards regression analysis.

\section{Statistical analysis}

The statistical analysis was performed in R software (version 3.5.3) and SPSS statistics (IBM, ver. 26.0). The normality of data distribution was assessed with the Shapiro-Wilk test. Continuous variables (tumor size, follow-up time and radiomics signature) were presented as medians (interquartiles). Categorical variables (gender, age, smoking history, pack-year, histological grade, CEA status, Ki$67 \mathrm{LI}$, pathological TNM stage, EGFR mutation status and therapy) were presented as numbers (percentages). 
The continuous variables between two groups (training cohort and validation cohort; high RS subgroup and low RS subgroup; patients receiving surgery alone and surgery plus ACT) were compared using the Mann-Whitney U test. For categorical variables, comparisons of two groups were performed using the Chi-square test or Fisher's exact test. The DFS rates of patients receiving surgery alone and surgery plus ACT were compared with the Chi-square test or Fisher's exact test. The variables significantly associated with DFS in univariate Cox regression were then analyzed in multivariate Cox regression with a method of forward stepwise to assess whether variables were independently associated with DFS. The two-sided $\mathrm{P}$ value less than 0.05 indicated a significant difference.

\section{Results}

\section{Baseline characteristics}

All baseline characteristics between training cohort $(n=186)$ and validation cohort $(\mathrm{n}=124)$ were similarly distributed as revealed in Table 1. Of the 310 eligible patients, 156 (50.3\%) were male patients and the median age was 59 years. Totally, $67(21.6 \%)$ patients were subjected to a recurrence or death: locoregional relapse and lung metastasis occurred in seven and thirty-one patients, respectively; nine, seven, three and two patients experienced brain, bone, liver, and pleural metastases, respectively; four patients experienced cervical or abdominal lymph node metastases; concurrent lung and brain metastases occurred in three patients; one patient died from primary hepatic carcinoma. The range of DFS of all patients was 4 to 84 months and the median (inter-quartile) DFS was 45.5 (36.0-54.0) months. The DFS of patients in training cohort [median (inter-quartile): $43.5(36,54)]$ and validation cohort $[48(36,54.75)]$ were balanced $(\mathrm{P}=0.834)$. In total, $226(72.9 \%)$ patients underwent surgery alone and $84(27.1 \%)$ patients received postsurgical ACT.

\section{Radiomics signature construction and validation}

A total of 851 radiomics features were extracted from the delineated three-dimensional volume of interests (Table S1). After excluding the radiomics features with ICC $<0.80,704$ features were retained. The redundant features with Spearman correlation coefficient $>0.7$ were then removed and the remaining 42 features were reserved to be normalized by $\mathrm{Z}$-score and selected by LASSO Cox regression algorithm. Eventually, thirteen nonzero coefficient features were used to construct radiomics signature (Figure $2 A, 2 B$ ) and their regression coefficients were depicted in Figure 2C. The detailed formula for calculating the Rad-score was showed in Appendix 2. The optimal threshold of Rad-score generated by the X-tile was 0.169 , which classified patients into high Rad-score and low Rad-score subgroup. Patients with high Rad-score $(>0.169)$ had poorer survival than those with low Radscore $(\leq 0.169)$. Kaplan-Meier curves for two subgroups were significantly separate in training cohort (HR: 13.45; 95\% CI: $5.25,34.42 ; \mathrm{P}<0.001)$ and a significant survival difference was also observed in validation cohort [HR: 6.51; 95\% CI: 2.64, 16.08; $\mathrm{P}<0.001$; Figure $3 A(A 1, A 2)]$. When all patients were stratified by gender, age level, smoking history, CEA status, Ki-67 LI and tumor size $(4 \mathrm{~cm})$, the survival differences between two subgroups were also significant (all $\mathrm{P}<0.05$; Figure $3 B-3 G$ ). The radiomics signature achieved a C-index of 0.779 (95\% CI: 0.719 , $0.840)$ in training cohort and 0.801 (95\% CI: $0.713,0.890)$ in validation cohort.

\section{Construction and performance of clinical nomogram and clinical-radiomics nomogram}

To avoid multicollinearity in regression equation, we excluded pathological TNM stage from regression analysis due to a strong correlation with tumor size. EGFR mutation status did not be involved in analysis given the excessive missing records in gene mutation detection. Finally, histological grade (high grade: HR: 6.420; 95\% CI: 2.575, 16.006; $\mathrm{P}<0.001$; intermediate grade: HR: 2.113; 95\% CI: 0.887, 5.003; $\mathrm{P}=0.091$; low grade: reference) and tumor size (HR: 1.687; 95\% CI: 1.395, 2.040; $\mathrm{P}<0.001$ ) as the independent clinical risk predictors in multivariate Cox regression analysis were incorporated to construct the clinical nomogram. Histological grade (high grade: HR: 3.343; 95\% CI: 1.300, 8.595; $\mathrm{P}=0.012$; intermediate grade: HR: 1.554 ; $95 \%$ CI: $0.643,3.756$; $\mathrm{P}=0.328$; low grade: reference), tumor size (HR: 1.295; 95\% CI: 1.038, 1.615; $\mathrm{P}=0.022$ ) and radiomics signature (HR: $3.207 ; 95 \%$ CI: 2.105, 4.884; $\mathrm{P}<0.001)$ were included to construct the clinical-radiomics nomogram (Table 2, Figure 4A,4B). In training cohort, the clinical-radiomics nomogram (C-index: 0.822; 95\% CI: $0.769,0.876)$ showed an improvement in predictive performance over clinical nomogram (C-index: 0.792; 95\% CI: $0.733,0.850)$ but it did not reach statistical significance $(\mathrm{P}=0.458)$. The consistent results were observed in validation cohort $(\mathrm{C}$-index for clinical-radiomics 
Table 1 The baseline characteristics of patients with node-negative, early-stage lung adenocarcinoma in training cohort and validation cohort

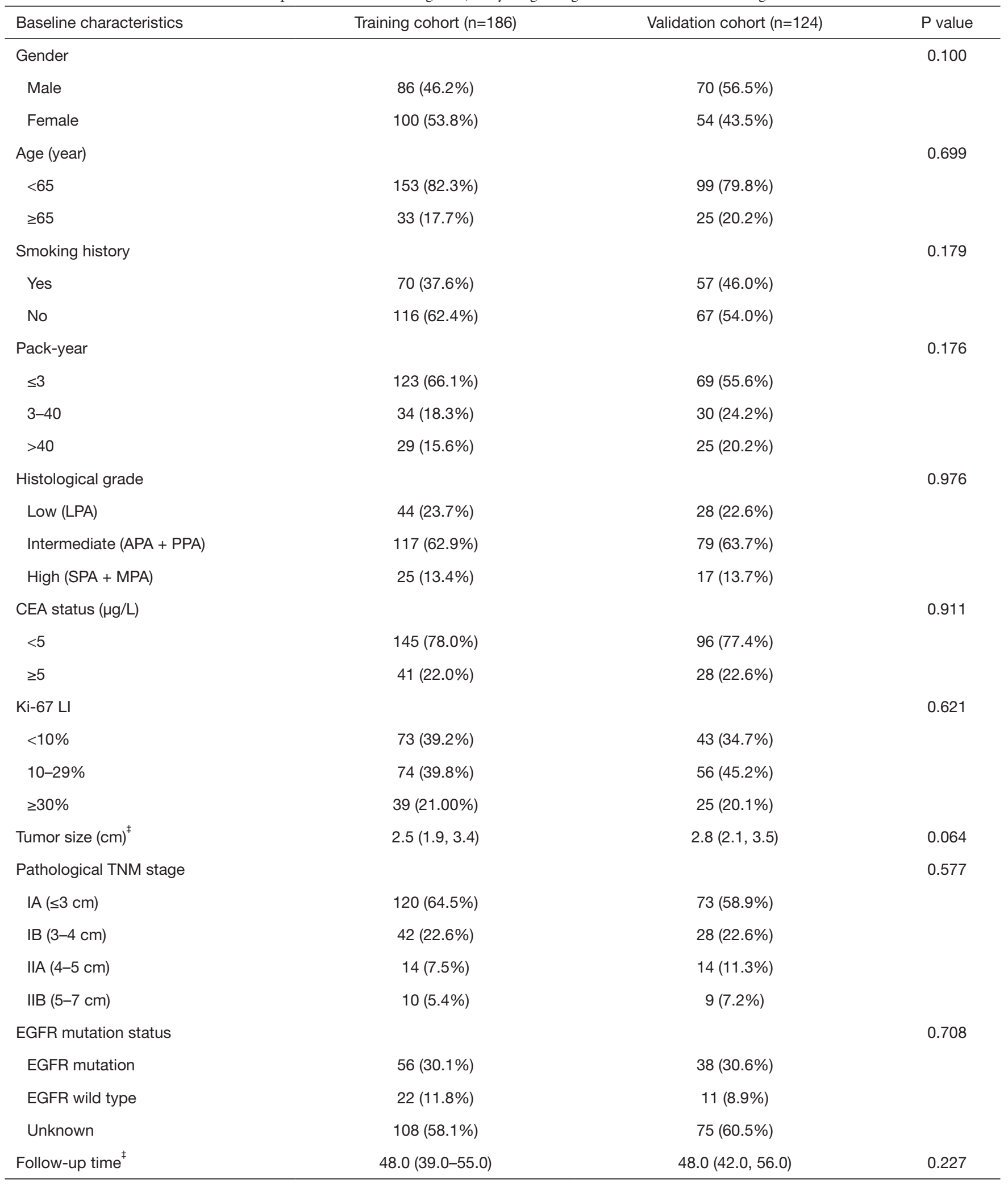

Table 1 (continued) 
Table 1 (continued)

\begin{tabular}{lcc}
\hline Baseline characteristics & Training cohort $(\mathrm{n}=186)$ & Validation cohort $(\mathrm{n}=124)$ \\
\hline Therapy & & $\mathrm{P}$ value \\
Surgery alone & $138(74.2 \%)$ & $88(71.0 \%)$ \\
Surgery plus ACT & $48(25.8 \%)$ & $36(29.0 \%)$ \\
\hline
\end{tabular}

Unless otherwise stated, data were presented as numbers (percentages) and compared using the Chi-square test. ${ }^{\ddagger}$, data were presented as medians (inter-quartiles) and compared using the Mann-Whitney $U$ test. Histological grade, Histological grade is the prognostic classification of lung adenocarcinoma predominant growth patterns, which separates invasive lung adenocarcinoma into three prognostic grades: low grade, intermediate grade and high grade; LPA, lepidic predominant adenocarcinoma; APA, acinar predominant adenocarcinoma; PPA, papillary predominant adenocarcinoma; SPA, solid predominant adenocarcinoma; MPA, micropapillary predominant adenocarcinoma; CEA, carcinoembryonic antigen; LI, labeling index; ACT, adjuvant chemotherapy; EGFR, epidermal growth factor receptor; TNM, tumor-node-metastasis.
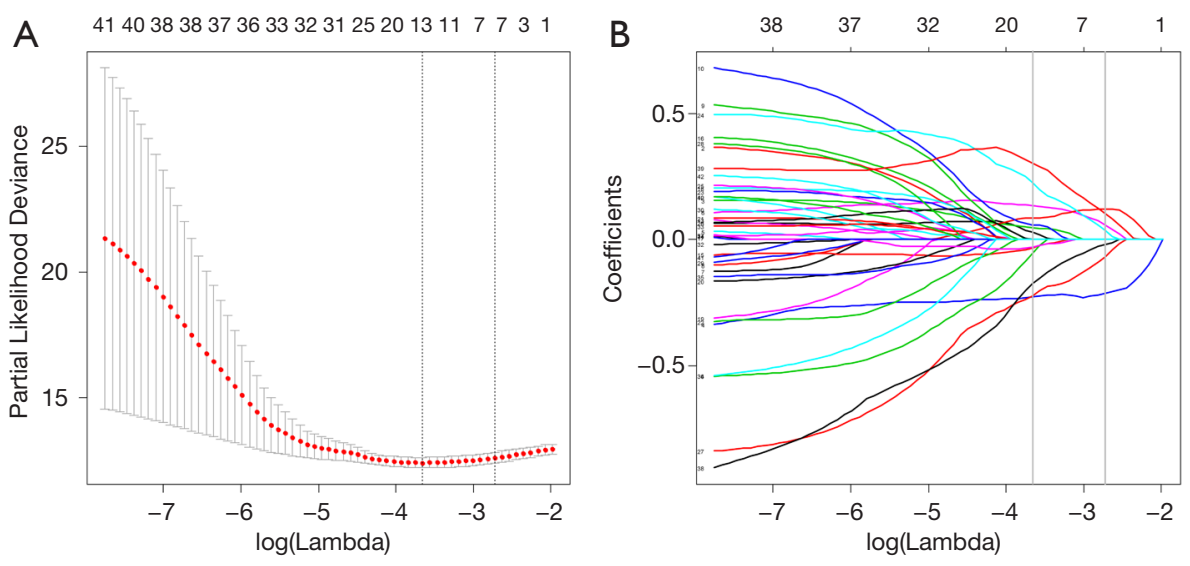

C

original glcm Imc2 wavelet.HHL glcm Correlation wavelet. $\mathrm{HHH}$ ngtdm Contrast wavelet. HHH glcm MCC wavelet.LHH firstorder Median wavelet.HHH gldm SmallDependenceLowGrayLevelEmphasis original shape Elongation original firstorder Kurtosis wavelet.LHL firstorder Mean wavelet.LLH glrlm LongRunHighGrayLevelEmphasis wavelet.LLH glcm ClusterShade wavelet.HLH glszm ZoneEntropy. wavelet.LLL firstorder 90Percentile.

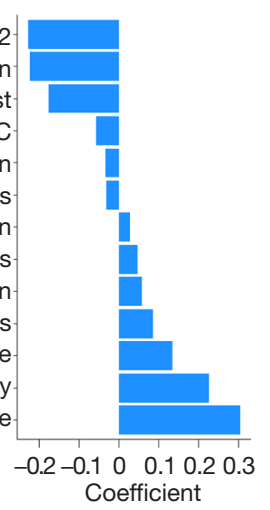

Figure 2 CT-derived radiomics feature selection using the LASSO Cox regression algorithm. (A) The optimal tuning parameter ( $\lambda$ ) was selected from LASSO Cox regression analysis with 10-fold cross validation. The curve of partial likelihood deviation versus log ( $\lambda$ ) was plotted. The optimal $\lambda$ value was 0.026 at the $\log (\lambda)$ value of -3.657 where the left dotted vertical lines intersected $x$-axis; (B) the optimal radiomics features set with non-zero coefficients were obtained at the $\log (\lambda)$ values of -3.657 where the left solid vertical lines intersected $\mathrm{x}$-axis; (C) the respective LASSO coefficients for the thirteen radiomics features with non-zero coefficients. CT, computer tomography; LASSO, least absolute shrinkage and selection operator. 


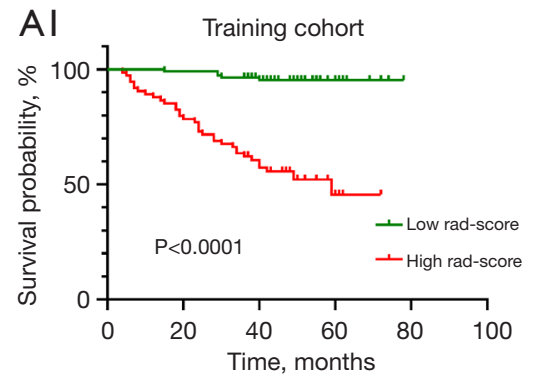

B2
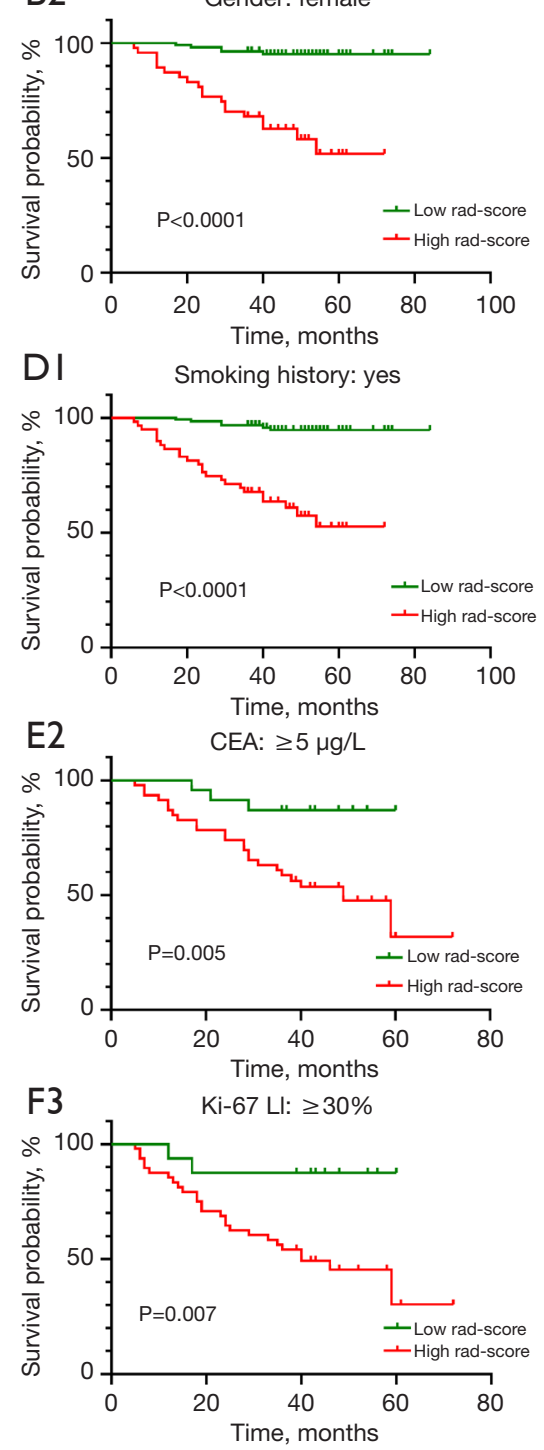

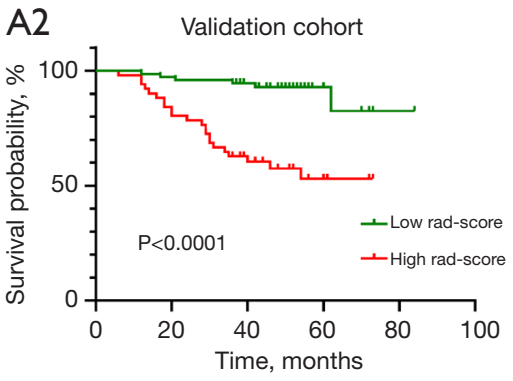

CI

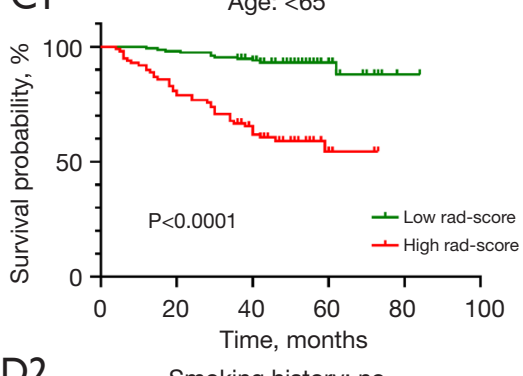

\section{D2}
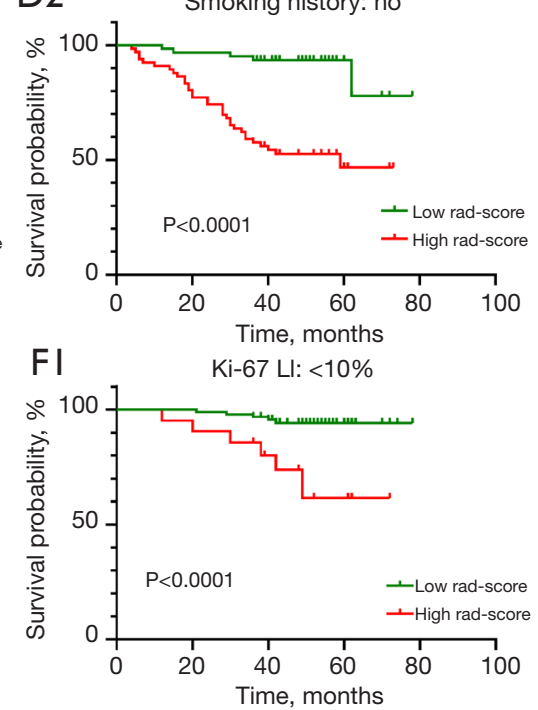

GI

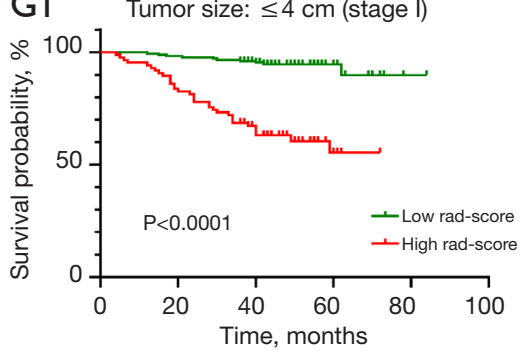

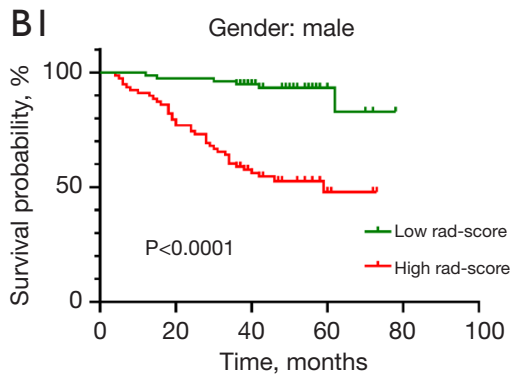

C2

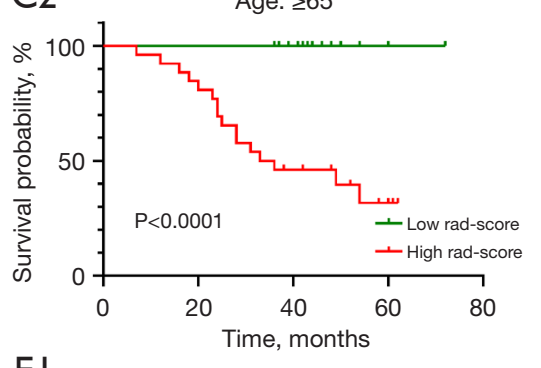

EI CEA: $<5 \mu \mathrm{g} / \mathrm{L}$
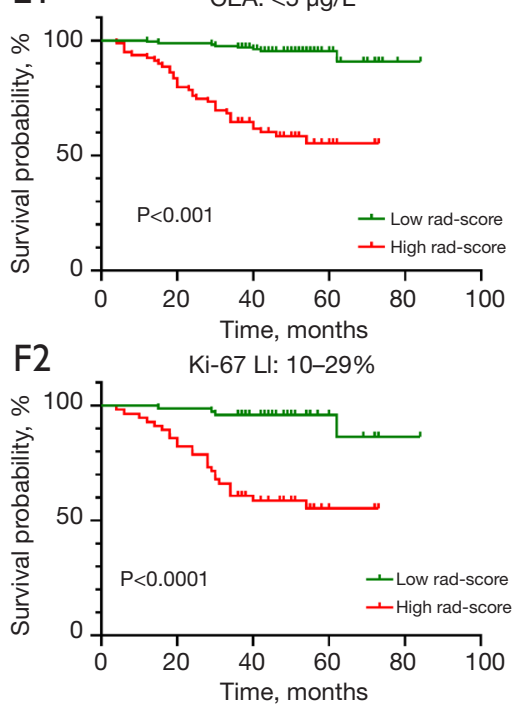

G2

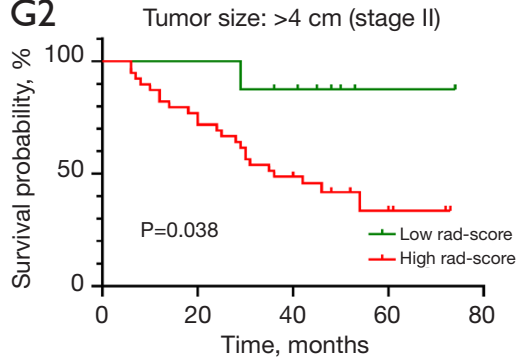

Figure 3 The Kaplan-Meier curves for high Rad-score subgroup versus low Rad-score subgroup. The Kaplan-Meier curves for high Radscore subgroup versus low Rad-score subgroup based on radiomics signature in training cohort (A1) and validation cohort (A2); the KaplanMeier curves in all patients stratified by gender, age level, smoking history, CEA status, Ki-67 LI, and tumor size (4 cm) (B1-G2). CEA, carcinoembryonic antigen; LI, labeling index. 
Table 2 Univariate and multivariate analysis of clinical characteristics and radiomics signature in patients with node-negative, early-stage lung adenocarcinoma

\begin{tabular}{|c|c|c|c|c|}
\hline Characteristics & \multicolumn{2}{|c|}{ Univariate analysis } & \multicolumn{2}{|c|}{ Multivariate analysis } \\
\hline Gender & & 0.014 & & 0.569 \\
\hline Female & Reference & & Reference & \\
\hline Male & $1.867(1.133,3.076)$ & & $1.246(0.585,2.657)$ & \\
\hline$\leq 65$ & Reference & & - & \\
\hline$>65$ & $1.417(0.808,2.485)$ & & - & \\
\hline Smoking status & & 0.01 & & 0.547 \\
\hline No & Reference & & Reference & \\
\hline$\leq 3$ & Reference & 0.007 & Reference & 0.634 \\
\hline $3-40$ & $1.384(0.738,2.595)$ & 0.311 & $0.465(0.096,2.258)$ & 0.342 \\
\hline$>40$ & $2.523(1.455,4.378)$ & 0.001 & $0.522(0.110,2.479)$ & 0.414 \\
\hline \multicolumn{5}{|l|}{ Histological grade } \\
\hline Low & Reference & $<0.001$ & Reference & 0.007 \\
\hline Intermedia & $2.528(1.066,5.994)$ & 0.035 & $1.554(0.643,3.756)$ & 0.328 \\
\hline High & $10.483(4.264,25.770)$ & $<0.001$ & $3.343(1.300,8.595)$ & 0.012 \\
\hline CEA status $(\mu \mathrm{g} / \mathrm{L})$ & & $<0.001$ & & 0.812 \\
\hline$\geq 30 \%$ & $6.209(3.084,12.500)$ & $<0.001$ & $1.240(0.488,3.153)$ & 0.651 \\
\hline Tumor size $(\mathrm{cm})$ & $1.823(1.529,2.172)$ & $<0.001$ & $1.295(1.038,1.615)$ & 0.022 \\
\hline Therapy & & 0.243 & & - \\
\hline Surgery alone & Reference & & - & \\
\hline Surgery plus ACT & $0.704(0.391,1.269)$ & & - & \\
\hline Radiomics signature & $4.639(3.297,6.527)$ & $<0.001$ & $3.207(2.105,4.884)$ & $<0.001$ \\
\hline
\end{tabular}

CEA, carcinoembryonic antigen; HR, hazard ratio; Cl, confidence interval; LI, labeling index; ACT, adjuvant chemotherapy. 


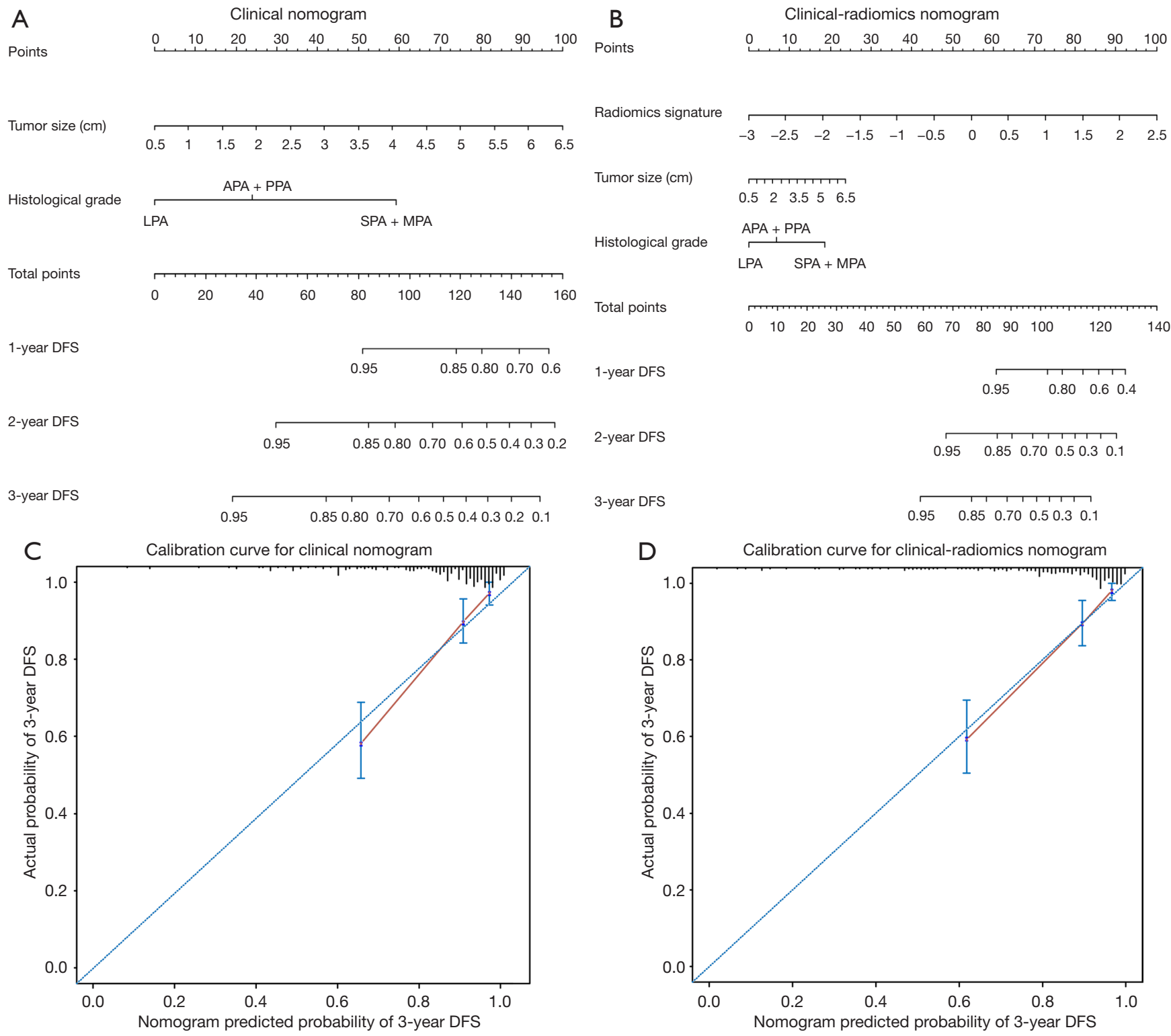

Figure 4 The constructed nomograms with their corresponding calibration curves. The clinical nomogram (A) and clinical-radiomics nomogram (B) for predicting the probabilities of 1-, 2-, and 3-year DFS. The calibration curves for clinical nomogram (C) and clinicalradiomics nomogram (D) depict the agreement between the predicted probabilities of 3-year DFS with the nomogram and the actual observed probabilities of 3-year DFS. The x-axis indicates the estimated probabilities of 3-year DFS with the nomogram and the y-axis indicates the actual observed probabilities of 3-year DFS. The diagonal dotted line plots the nomogram predicted results fitting perfectly the actual observed results. The solid line plots the performance of the constructed nomogram. The solid line more closely fitting the diagonal dotted line represents a better predictive performance. APA, acinar predominant adenocarcinoma; PPA, papillary predominant adenocarcinoma; SPA, solid predominant adenocarcinoma; MPA, micropapillary predominant adenocarcinoma; LPA, lepidic predominant adenocarcinoma; DFS, disease free survival.

nomogram: $0.802 ; 95 \%$ CI: $0.716,0.888$; C-index for clinical nomogram: 0.792; 95\% CI: $0.733,0.850 ; \mathrm{P}=0.654$ ). However, with the incorporation of radiomics signature into clinical-radiomics nomogram, the predictive accuracy significantly improved compared to clinical nomogram in terms of IDI (0.082; 95\% CI: 0.002, 0.178; $\mathrm{P}=0.040)$ and 


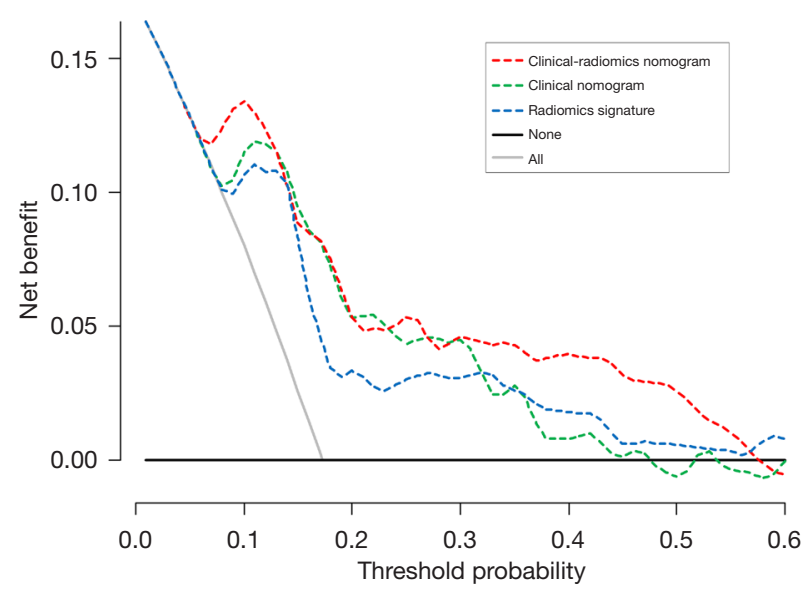

Figure 5 Decision curve analysis of different models. The x-axis represents the threshold probability. The y-axis represents the net benefits in comprehensive consideration of insufficient treatment (false negative) and excessive treatment (false positive) across the threshold probability of 0-0.6. Decision curve analysis reveals that clinical-radiomics nomogram had a higher net benefit than the radiomics signature or clinical nomogram in clinical practice.

NRI $(0.261 ; 95 \%$ CI: $0.002,0.485 ; \mathrm{P}=0.040)$ in training cohort. The incremental benefit with the integration of radiomics signature was also significant in validation cohort (IDI $=0.114 ; 95 \%$ CI: $0.026,0.209 ; \mathrm{P}=0.007)$ though NRI was not but approximately significant $(0.395 ; 95 \%$ CI: $-0.008,0.613, \mathrm{P}=0.053)$. Furthermore, the calibration curve showed that the probabilities of 3-year DFS estimated with clinical-radiomics nomogram were more agreement with actual observed probabilities (Figure 4C,4D). Decision curve analysis showed that clinical-radiomics nomogram had a higher net benefit than radiomics signature or clinical nomogram across the vast majority of the threshold probability of 0-0.6 (Figure 5).

\section{Predictive ability of clinical-radiomics nomogram in ACT benefit}

The RS for clinical-radiomics nomogram was calculated for each patient with the following formula: $\mathrm{RS}=0.0 \times$ 0 (if histological grade: low) $+0.441 \times 1$ (if histological grade: intermediate) $+1.207 \times 1$ (if histological grade: high) $+0.258 \times$ tumor size $+1.165 \times$ radiomics signature. We stratified patients into high RS and low RS subgroup by the optimal threshold of 1.570 . The survival differences between high RS subgroup $(\geq 1.570)$ and low RS subgroup $(<1.570)$ was significant both in training cohort (HR: 15.45;
95\% CI: 6.03, 39.57; $\mathrm{P}<0.001)$ and validation cohort (HR: 5.49; 95\% CI: 2.33, 12.94; $\mathrm{P}<0.001$; Figure 6A,6B). Table 3 indicated that male smokers with larger pack-year, CEA $\geq 5 \mu \mathrm{g} / \mathrm{L}$, higher histological grade and Ki-67 LI, larger tumor size, EGFR wild type and receiving postsurgical ACT were prone to harbor high RS. Furthermore, patients with EGFR mutation harbored significantly lower RS than those with EGFR wild type $(\mathrm{P}=0.013)$. One-year, two-year and three-year DFS rates of patients in low RS subgroup were significantly higher than those in high RS subgroup (all $\mathrm{P}<0.001 ;$ Table 4).

In this study, patients receiving postsurgical ACT were prone to harbor higher Ki-67 LI and radiomics signature, larger tumor size, and be in high RS subgroup (Table S2). Interestingly, ACT was significantly associated with survival benefit in high RS subgroup (HR: 0.489; 95\% CI: 0.262, 0.911; $\mathrm{P}=0.024)$, whereas ACT was not beneficial to patients in low RS subgroup (HR: 0.324; 95\% CI: 0.042, 2.510; $\mathrm{P}=0.281$; Figure $6 C, 6 D)$. Furthermore, 3-year DFS rates for patients treated with surgery alone $(53.2 \%)$ and surgery plus ACT (76.2\%) were incomparable in high RS subgroup $(\mathrm{P}=0.014)$, but no significant beneficial effect of ACT on DFS rates was observed in low RS subgroup (all $\mathrm{P}>0.05$; Table 4). These findings suggested that the clinicalradiomics nomogram may confer a role in identifying patients who were likely to acquire benefit from ACT.

\section{Discussion}

In this study, we developed and validated a radiomics signature reliably prognostic of DFS for node-negative, early-stage ADC. This noninvasive radiomics signature, readily obtained from routine presurgical thoracic-CT images, was independently associated with DFS, even after adjustment for clinical characteristics. Moreover, the radiomics signature exhibited an incremental value with respect to clinical risk predictors of tumor size and histological grade in individualized risk prediction. The integrated clinical-radiomics nomogram not only exhibited a desirable performance in risk stratification but harbored the potential to identify potential candidates for ACT in patients with node-negative, early-stage ADC.

The thirteen-feature based radiomics signature yielded a C-index of 0.801 in validation cohort, which exhibited a better prognostic accuracy than a previous study by Choe et al. (C-index: 0.734) in predicting DFS for ADC (25). An explanation may be that radiomics signature in this study was derived from feature sets including wavelet-transformed 

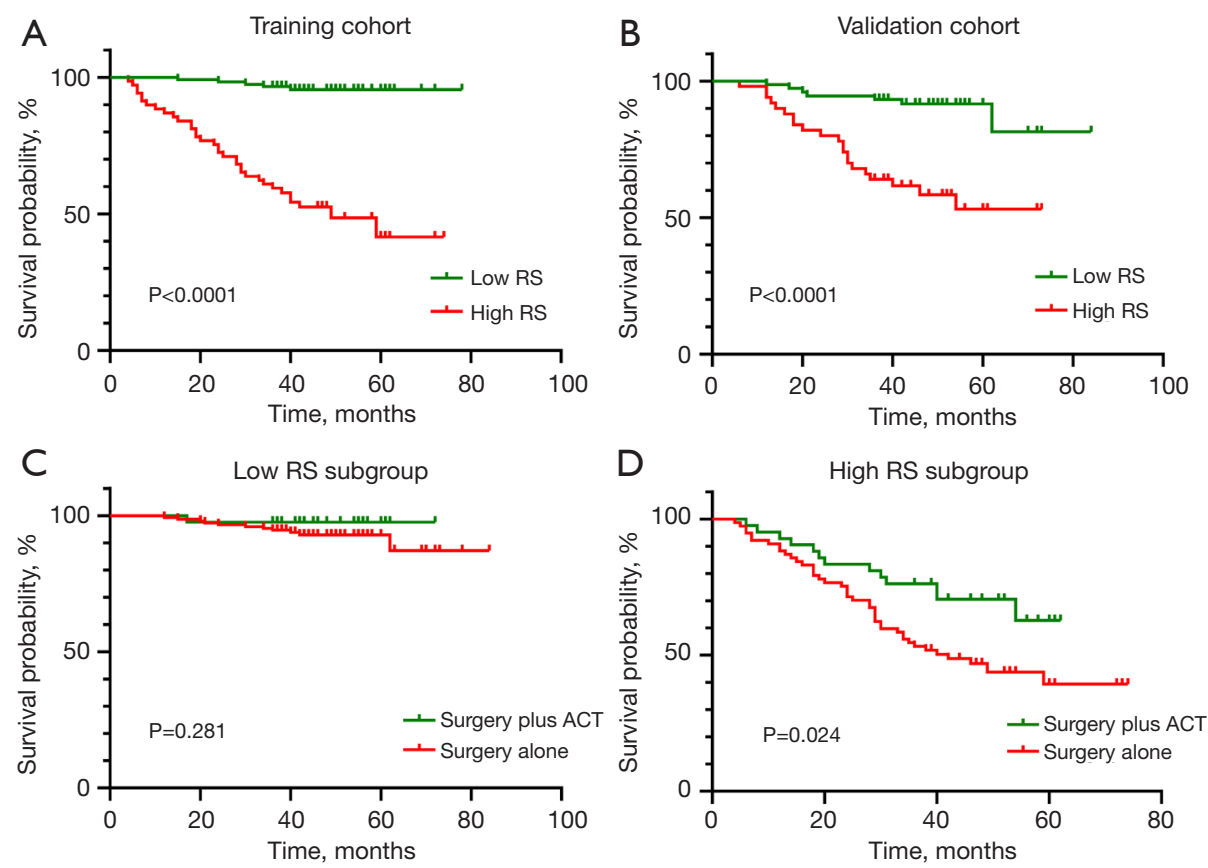

Figure 6 Kaplan-Meier curves in clinical-radiomics nomogram. DFS of patients with high RS versus patients with low RS based on clinicalradiomics nomogram in training cohort (A) and validation cohort (B); DFS of all patients treated with surgery plus ACT versus treated with surgery alone in low RS subgroup (C) and high RS subgroup (D). DFS, disease free survival; RS, risk score; ACT, adjuvant chemotherapy.

features, which offered comprehensive spatial and frequency distribution of voxel-grey levels by decomposing images into eight volumetric images with a high-pass or low-pass filter $(26,27)$. The benefit of wavelet-transformed features in enhancing model performance was also demonstrated by previous studies $(27,28)$. Furthermore, the incorporation of radiomics signature into clinical-radiomics nomogram showed a substantial improvement compared to clinical nomogram in terms of NRI and IDI. The clinical-radiomics nomogram exhibited a better calibration as well as a superior clinical utility from decision curve analysis, which further lent support to the incremental value of radiomics signature over clinical risk predictors for predicting prognosis in node-negative, early-stage ADC.

In the developed radiomics signature, the best performing feature "first order_90th Percentile", which probably indicates intratumor solid component, consistently revealed the prognostic potential for DFS in a prior study (25). The other predominant features such as glcm_Imc2, and glszm_ZoneEntropy, glcm_Correlation, ngtdm_Contrast, and $\mathrm{glcm}$ - ClusterShade were derived from original and multiscale wavelets families, respectively. These texture features could quantify intratumor heterogeneity and reflect tumor phenotype characteristics at radiological level $(29,30)$, which have been used as prognosticators for ADC (31-33). Radiogenomics provided insight into the underlying biological mechanism of radiomics approach and revealed the association of genetic phenotype and biological behavior with radiomics features. Series studies reported that prognostic radiomics features were closely associated with cell atypia, angiogenesis or metabolic pathways to facilitate tumor growth $(34,35)$. Given that, it is understandable that the synthetic radiomics signature could take on the prognostic potential.

For node-negative NSCLC, tumor size was the predominant determinant of survival as well as the reference criterion for directing ACT decision-making (10). However, many studies reported controversial results regarding the selection of candidates for ACT $(10,12,36,37)$. Strauss et al. initially reported that ACT improved DFS and overall survival in patients with node-negative tumors $4 \mathrm{~cm}$ or greater in a randomized clinical trial (36), whereas Morgensztern et al. suggested a survival benefit associated with ACT in all node-negative tumors $3-4 \mathrm{~cm}$ (37). In a retrospective study, ACT exhibited a survival advantage in high-risk (micropapillary predominant) patients with nodenegative small-size $(\leq 3 \mathrm{~cm})$ lung ADC (12). Contrary to this, a more recent large-sample study failed to observe a 
Table 3 The distribution of clinical characteristics and radiomics signature in high RS and low RS subgroups based on clinical-radiomics nomogram

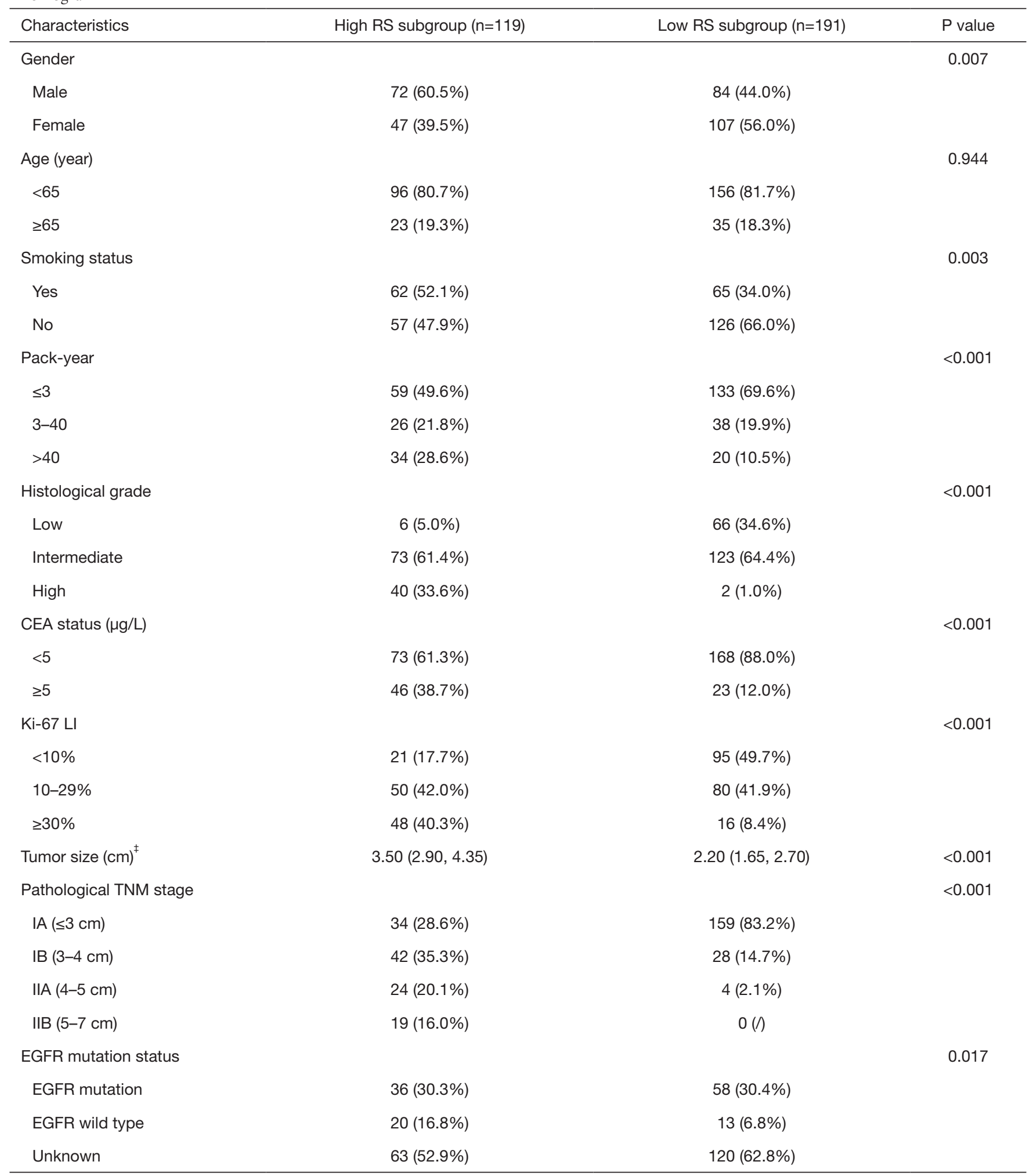

Table 3 (continued) 
Table 3 (continued)

\begin{tabular}{|c|c|c|c|}
\hline Characteristics & High RS subgroup $(n=119)$ & Low RS subgroup $(n=191)$ & $P$ value \\
\hline Therapy & & & 0.010 \\
\hline Surgery alone & $77(64.7 \%)$ & $149(78.0 \%)$ & \\
\hline Surgery plus ACT & $42(35.3 \%)$ & $42(22.0 \%)$ & \\
\hline
\end{tabular}

Unless otherwise stated, data were presented as numbers (percentages) and compared using the Chi-square test or Fisher's exact test. ${ }^{\ddagger}$, data were presented as medians (inter-quartiles) and compared using the Mann-Whitney U test. RS, risk score; CEA, carcinoembryonic antigen; LI, labeling index; TNM, tumor-node-metastasis; EGFR, epidermal growth factor receptor; ACT, adjuvant chemotherapy.

Table 4 The disease-free survival rates at 1-, 2-, and 3-year of patients treated with surgery versus surgery plus ACT in high/low RS subgroup according to clinical-radiomics nomogram

\begin{tabular}{|c|c|c|c|c|c|c|c|c|c|}
\hline \multirow{2}{*}{$\begin{array}{l}\text { DFS rate, } \\
n / N(\%)\end{array}$} & \multicolumn{4}{|c|}{ High RS subgroup } & \multicolumn{4}{|c|}{ Low RS subgroup } & \multirow{2}{*}{ P value* } \\
\hline & Total $(\mathrm{N}=119)$ & Surgery $(\mathrm{N}=77$ ) & $\begin{array}{l}\text { Surgery plus ACT } \\
\qquad(n=42)\end{array}$ & P value ${ }^{\dagger}$ & Total $(N=191)$ & Surgery $(N=149)$ & $\begin{array}{l}\text { Surgery plus ACT } \\
\qquad(\mathrm{N}=42)\end{array}$ & $P$ value ${ }^{\ddagger}$ & \\
\hline At 1-year & $\begin{array}{l}107 / 119 \\
(89.9 \%)\end{array}$ & 68/77 (88.3\%) & $39 / 42$ (92.9\%) & 0.639 & $\begin{array}{l}190 / 191 \\
(99.5 \%)\end{array}$ & 148/149 (99.3\%) & $42 / 42(100 \%)$ & 1.000 & $<0.001$ \\
\hline At 3-year & $\begin{array}{l}73 / 119 \\
(61.3 \%)\end{array}$ & $41 / 77$ (53.2\%) & $32 / 42(76.2 \%)$ & 0.014 & $\begin{array}{l}182 / 191 \\
(95.3 \%)\end{array}$ & $141 / 149$ (94.6\%) & $41 / 42(97.6 \%)$ & 0.693 & $<0.001$ \\
\hline
\end{tabular}

All comparisons were performed using the Chi-square test or Fisher's exact test. ${ }^{\dagger}$, the comparison of DFS rates between patients treated with surgery and surgery plus ACT in high RS subgroup; ${ }^{\ddagger}$, the comparison of DFS rates between patients treated with surgery and surgery plus ACT in low RS subgroup; *, the comparison of DFS rates between patients in high RS subgroup and low RS subgroup. ACT, adjuvant chemotherapy; RS, risk score; DFS, disease free survival.

beneficial effect of ACT in patients with $3 \mathrm{~cm}$ or smaller irrespective of the presence of clinicopathological risk factors (10). Because of these controversial findings and the non-trivial recurrence for patients with early-stage NSCLC, it is imperative to exploit effective methods to refine the indication for ACT. Currently, several studies have applied the radiomics approach to predict therapeutic response in various types of cancer (38-40). Jiang et al. established and validated a CT-derived radiomics signature to evaluate the immune microenvironment and predict immunotherapeutic effect in gastric cancer (38). Montemezzi et al. retrospectively analyzed the magnetic resonance images of 60 patients with breast cancer and found that radiomics features substantially improved performance of model in predicting complete response to neoadjuvant chemotherapy (39). In this study, we stratified patients into different subgroups based on RS for clinical-radiomics model.
We found that male smokers with larger pack-year, CEA $\geq 5 \mu \mathrm{g} / \mathrm{L}$, higher histological grade and Ki-67 LI, larger tumor size and EGFR wild type were likely to harbor high RS. The patients with high RS significantly acquired benefit from ACT whereas no distinct survival benefit from ACT was observed in patients with low RS, suggesting that the clinical-radiomics model may confer a role in identifying potential beneficiaries of ACT among node-negative, earlystage ADC. On account of the retrospective nature of data acquisition, the time to initiate ACT following surgery and treatment regimens for individuals were hard to be uniform. Therefore, prospective, randomly-controlled trials are warranted to confirm the clinical utility of clinical-radiomics model in predicting benefit of ACT. This exploratory study might not direct therapeutic decision-making instantly, but the developed model could provoke thoughts on risk stratification by integrating routine clinicopathological data 
and readily-accessed imaging predictors, not only assisting in guiding appropriate patients in benefiting from ACT but in avoiding toxic effects and side reaction of ACT in potential no-responders.

There are several limitations to the present study. First, the reproducibility of radiomics features is an ongoing issue that we need to address. Multi-vendor CT scanners and different acquisition parameters in external datasets were required to verify the reproducibility of the developed radiomics signature. Second, considering this radiomics feature extraction from contrast-enhanced CT images, the variation of radiomics signature in non-enhanced CT images should be tested in further work. Third, the analysis on the development of radiomics signature prognostic of overall survival should be supplemented in future research. Last, gene mutation status was excluded from cox regression analysis due to the excessive missing records in gene mutation detection. The prognostic role of genetic mutation and the association of radiomics signature with genetic mutation as well as other molecular profiles should be explored in future work.

In conclusion, radiomics signature offered a complementary role to clinical risk predictors of tumor size and histological grade in predicting prognosis in node-negative, early-stage ADC. The clinical-radiomics nomogram has the potential to conduct risk stratification and identify the potential beneficiaries of ACT among patients with node-negative, early-stage ADC. On the premise of validation by large sample-size external cohorts, the approach of radiomics signature in combination with clinical risk predictors is expected to be a helpful tool for clinicians to make up individualized therapeutic schedule.

\section{Acknowledgments}

We would like to acknowledge Wei $\mathrm{Wu}$ for the language revision of this manuscript.

Funding: This study was supported by the National Natural Science Foundation of China (No. 81873889).

\section{Footnote}

Reporting Checklist: The authors have completed the TRIPOD reporting checklist. Available at https://jtd. amegroups.com/article/view/10.21037/jtd-21-1520/rc

Data Sharing Statement: Available at https://jtd.amegroups. com/article/view/10.21037/jtd-21-1520/dss
Conflicts of Interest: All authors have completed the ICMJE uniform disclosure form (available at https://jtd.amegroups. com/article/view/10.21037/jtd-21-1520/coif). The authors have no conflicts of interest to declare.

Ethical Statement: The authors are accountable for all aspects of the work in ensuring that questions related to the accuracy or integrity of any part of the work are appropriately investigated and resolved. The study was conducted in accordance with the Declaration of Helsinki (as revised in 2013). This study was approved by the Institutional Ethics Committee of Tongji Hospital (No. TJIRB-20180615). Individual consent for this retrospective analysis was waived.

Open Access Statement: This is an Open Access article distributed in accordance with the Creative Commons Attribution-NonCommercial-NoDerivs 4.0 International License (CC BY-NC-ND 4.0), which permits the noncommercial replication and distribution of the article with the strict proviso that no changes or edits are made and the original work is properly cited (including links to both the formal publication through the relevant DOI and the license). See: https://creativecommons.org/licenses/by-nc-nd/4.0/.

\section{References}

1. Bray F, Ferlay J, Soerjomataram I, et al. Global cancer statistics 2018: GLOBOCAN estimates of incidence and mortality worldwide for 36 cancers in 185 countries. CA Cancer J Clin 2018;68:394-424.

2. Herbst RS, Morgensztern D, Boshoff C. The biology and management of non-small cell lung cancer. Nature 2018;553:446-54.

3. Hanagiri T, Baba T, So T, et al. Time trends of surgical outcome in patients with non-small cell lung cancer. J Thorac Oncol 2010;5:825-9.

4. Zhu CQ, Ding K, Strumpf D, et al. Prognostic and predictive gene signature for adjuvant chemotherapy in resected non-small-cell lung cancer. J Clin Oncol 2010;28:4417-24.

5. Scagliotti GV, Fossati R, Torri V, et al. Randomized study of adjuvant chemotherapy for completely resected stage I, II, or IIIA non-small-cell Lung cancer. J Natl Cancer Inst 2003;95:1453-61.

6. Arriagada R, Dunant A, Pignon JP, et al. Long-term results of the international adjuvant lung cancer trial evaluating adjuvant Cisplatin-based chemotherapy in resected lung 
cancer. J Clin Oncol 2010;28:35-42.

7. Douillard JY, Rosell R, De Lena M, et al. Adjuvant vinorelbine plus cisplatin versus observation in patients with completely resected stage IB-IIIA non-small-cell lung cancer (Adjuvant Navelbine International Trialist Association ANITA): a randomised controlled trial. Lancet Oncol 2006;7:719-27.

8. Winton T, Livingston R, Johnson D, et al. Vinorelbine plus cisplatin vs. observation in resected non-small-cell lung cancer. N Engl J Med 2005;352:2589-97.

9. Kris MG, Gaspar LE, Chaft JE, et al. Adjuvant systemic therapy and adjuvant radiation therapy for stage I to IIIA completely resected non-small-cell lung cancers: American Society of Clinical Oncology/Cancer Care Ontario Clinical Practice Guideline update. J Clin Oncol 2017;35:2960-74.

10. Pathak R, Goldberg SB, Canavan M, et al. Association of survival with adjuvant chemotherapy among patients with early-stage non-small cell lung cancer with vs without high-risk clinicopathologic features. JAMA Oncol 2020;6:1741-50.

11. Krivitsky TA, Wright GM, Al Zaidi M. A predictive model for identifying candidates for adjuvant chemotherapy based on recurrence risk profile of resected, node-negative (N0) non-small cell lung cancer. J Thorac Dis 2021;13:149-59.

12. Wang C, Yang J, Lu M. Micropapillary predominant lung adenocarcinoma in stage IA benefits from adjuvant chemotherapy. Ann Surg Oncol 2020;27:2051-60.

13. Chen D, Wang X, Zhang F, et al. Could tumor spread through air spaces benefit from adjuvant chemotherapy in stage I lung adenocarcinoma? A multi-institutional study. Ther Adv Med Oncol 2020;12:1758835920978147.

14. Woodard GA, Wang SX, Kratz JR, et al. Adjuvant chemotherapy guided by molecular profiling and improved outcomes in early stage, non-small-cell lung cancer. Clin Lung Cancer 2018;19:58-64.

15. Tsao MS, Aviel-Ronen S, Ding K, et al. Prognostic and predictive importance of $\mathrm{p} 53$ and RAS for adjuvant chemotherapy in non small-cell lung cancer. J Clin Oncol 2007;25:5240-7.

16. Muley T, Rolny V, He Y, et al. The combination of the blood based tumor biomarkers cytokeratin 19 fragments (CYFRA 21-1) and carcinoembryonic antigen (CEA) as a potential predictor of benefit from adjuvant chemotherapy in early stage squamous cell carcinoma of the lung (SCC). Lung Cancer 2018;120:46-53.

17. Filipits M, Pirker R. Predictive markers in the adjuvant therapy of non-small cell lung cancer. Lung Cancer
2011;74:355-63.

18. Hatt M, Le Rest CC, Tixier F, et al. Radiomics: data are also images. J Nucl Med 2019;60:38S-44S.

19. Gillies RJ, Kinahan PE, Hricak H. Radiomics: images are more than pictures, they are data. Radiology 2016;278:563-77.

20. Song SH, Park H, Lee G, et al. Imaging phenotyping using radiomics to predict micropapillary pattern within lung adenocarcinoma. J Thorac Oncol 2017;12:624-32.

21. Yoshizawa A, Sumiyoshi S, Sonobe M, et al. Validation of the IASLC/ATS/ERS lung adenocarcinoma classification for prognosis and association with EGFR and KRAS gene mutations: analysis of 440 Japanese patients. J Thorac Oncol 2013;8:52-61.

22. van Griethuysen JJM, Fedorov A, Parmar C, et al. Computational radiomics system to decode the radiographic phenotype. Cancer Res 2017;77:e104-7.

23. Pencina MJ, D'Agostino RB Sr, Steyerberg EW. Extensions of net reclassification improvement calculations to measure usefulness of new biomarkers. Stat Med 2011;30:11-21.

24. Fitzgerald M, Saville BR, Lewis RJ. Decision curve analysis. JAMA 2015;313:409-10.

25. Choe J, Lee SM, Do KH, et al. Outcome prediction in resectable lung adenocarcinoma patients: value of CT radiomics. Eur Radiol 2020;30:4952-63.

26. Chaddad A, Daniel P, Niazi T. Radiomics evaluation of histological heterogeneity using multiscale textures derived from 3D wavelet transformation of multispectral images. Front Oncol 2018;8:96.

27. Zhou J, Lu J, Gao C, et al. Predicting the response to neoadjuvant chemotherapy for breast cancer: wavelet transforming radiomics in MRI. BMC Cancer 2020;20:100.

28. Majeed Alneamy JS, A Hameed Alnaish Z, Mohd Hashim SZ, et al. Utilizing hybrid functional fuzzy wavelet neural networks with a teaching learning-based optimization algorithm for medical disease diagnosis. Comput Biol Med 2019;112:103348.

29. Lubner MG, Smith AD, Sandrasegaran K, et al. CT texture analysis: definitions, applications, biologic correlates, and challenges. Radiographics 2017;37:1483-503.

30. Aerts HJ, Velazquez ER, Leijenaar RT, et al. Decoding tumour phenotype by noninvasive imaging using a quantitative radiomics approach. Nat Commun 2014;5:4006.

31. Ravanelli M, Agazzi GM, Ganeshan B, et al. CT texture analysis as predictive factor in metastatic lung 
adenocarcinoma treated with tyrosine kinase inhibitors (TKIs). Eur J Radiol 2018;109:130-5.

32. Huang Y, Liu Z, He L, et al. Radiomics signature: a potential biomarker for the prediction of disease-free survival in early-stage (I or II) non-small cell lung cancer. Radiology 2016;281:947-57.

33. Sun Q, Huang Y, Wang J, et al. Applying CT texture analysis to determine the prognostic value of subsolid nodules detected during low-dose CT screening. Clin Radiol 2019;74:59-66.

34. Vaidya P, Bera K, Gupta A, et al. CT derived radiomic score for predicting the added benefit of adjuvant chemotherapy following surgery in stage I, II resectable non-small cell lung cancer: a retrospective multicohort study for outcome prediction. Lancet Digit Health 2020;2:e116-28.

35. Grossmann P, Stringfield O, El-Hachem N, et al. Defining the biological basis of radiomic phenotypes in lung cancer. Elife 2017;6:23421.

36. Strauss GM, Herndon JE 2nd, Maddaus MA, et al.

Cite this article as: $\mathrm{Ma} \mathrm{X}, \mathrm{Lv} \mathrm{W}$, Wang C, Tu D, Qiao J, Fan C, Niu J, Zhou W, Liu Q, Xia L. A potential biomarker based on clinical-radiomics nomogram for predicting survival and adjuvant chemotherapy benefit in resected node-negative, early-stage lung adenocarcinoma. J Thorac Dis 2022;14(1):117. doi: $10.21037 / j t d-21-1520$
Adjuvant paclitaxel plus carboplatin compared with observation in stage IB non-small-cell lung cancer: CALGB 9633 with the Cancer and Leukemia Group B, Radiation Therapy Oncology Group, and North Central Cancer Treatment Group Study Groups. J Clin Oncol 2008;26:5043-51.

37. Morgensztern D, Du L, Waqar SN, et al. Adjuvant chemotherapy for patients with T2N0M0 NSCLC. J Thorac Oncol 2016;11:1729-35.

38. Jiang $\mathrm{Y}$, Wang $\mathrm{H}, \mathrm{Wu}$ J, et al. Noninvasive imaging evaluation of tumor immune microenvironment to predict outcomes in gastric cancer. Ann Oncol 2020;31:760-8.

39. Montemezzi S, Benetti G, Bisighin MV, et al. 3T DCEMRI radiomics improves predictive models of complete response to neoadjuvant chemotherapy in breast cancer. Front Oncol 2021;11:630780.

40. Staal FCR, van der Reijd DJ, Taghavi M, et al. Radiomics for the prediction of treatment outcome and survival in patients with colorectal cancer: a systematic review. Clin Colorectal Cancer 2021;20:52-71. 


\section{Supplementary}

\section{Appendix 1}

\section{CT Image Scanning Parameters}

The patients were injected intravenously with contrast agent (Ultravist, Iopromide, Bayer Schering Pharma AG, Leverkusen, Germany) bolus at the speed of $2.5-3.5 \mathrm{~mL} / \mathrm{s}$ and a dosage of $1.1 \mathrm{~mL} / \mathrm{kg}$ of weight with the scan delay time of $30 \mathrm{~s}$. The lung scanning was performed from lung apex to lung basis during a breath-hold following inspiration. The CT acquisition parameters were field of view, $350 \times 350 \mathrm{~mm}$; matrix, $512 \times 512$; tube voltage, $120 \mathrm{kV}$; tube current, $300 \mathrm{~mA}$; pitch, 0.98 ; layer thickness, $5 \mathrm{~mm}$; reconstruction thickness and thickness interval, 1.25 and $1.25 \mathrm{~mm}$.

\section{Appendix 2}

Rad-score $=0.302808236 \times$ wavelet.LLL_firstorder_90Percentile $+(-0.227677645) \times$ original_glcm_Imc2 $+0.224944227 \times$ wavelet.HLH_glszm_ZoneEntropy $+(-0.222504561) \times$

wavelet.HHL_glcm_Correlation + $(-0.176735501) \times$ wavelet.HHH_ngtdm_Contrast + $0.134067339 \times$ wavelet.LLH_glcm_ClusterShade $+0.086051532 \times$ wavelet.LLH_glrlm_LongRunHighGrayLevelEmphasis + $0.05809147 \times$ wavelet.LHL_firstorder_Mean $+(-0.056029374) \times$ wavelet.HHH_glcm_MCC + 0.046175474 × original_firstorder_Kurtosis $+(-0.033741439) \times$ wavelet.LHH_firstorder_Median $+(-0.031014451) \times$ wavelet.HHH_gldm_SmallDependenceLowGrayLevelEmphasis + $0.026919459 \times$ original_shape_Elongation

Table S1 The category list of all extracted radiomics features

\begin{tabular}{lccc}
\hline Category & Original & Wavelet transformations & All \\
\hline Shape & 14 & - & 14 \\
First-order & 18 & 144 & 162 \\
GLCM & 24 & 192 & 216 \\
GLRLM & 16 & 128 & 128 \\
GLSZM & 16 & 112 & 144 \\
GLDM & 14 & 40 & 126 \\
NGTDM & 5 & 744 & 45 \\
Total & 107 & 851 & \\
\hline
\end{tabular}

GLCM, gray-level co-occurrence matrix; GLRLM, gray-level run-length matrix; GLSZM, gray-level size zone matrix; GLDM, gray-level dependence matrix; NGTDM, neighbouring gray-tone difference matrix. 
Table S2 The distribution of clinical characteristics, radiomics signature and risk level in all patients treated with surgery alone and surgery plus ACT

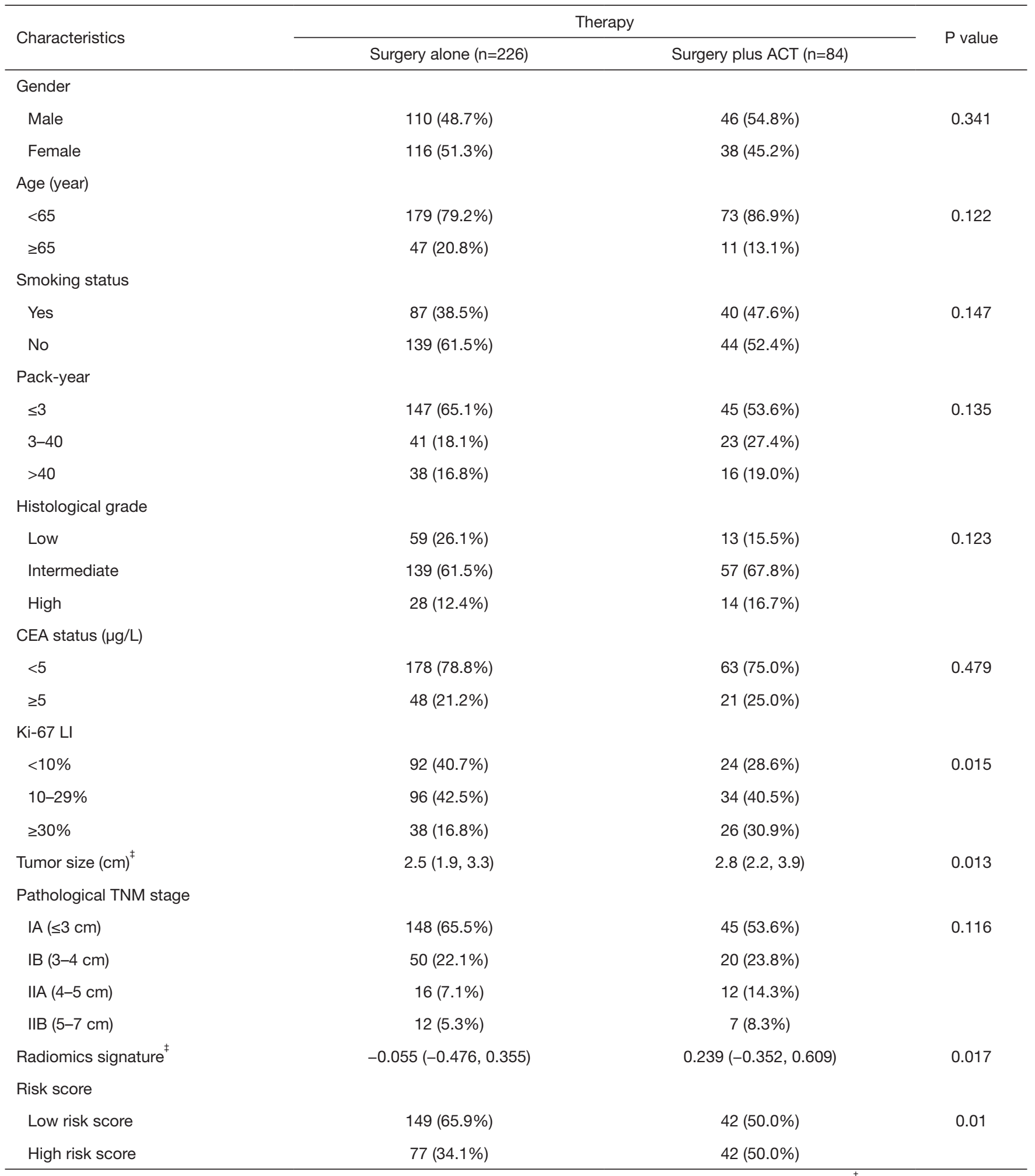

Unless otherwise stated, data were presented as numbers (percentages) and compared using the Chi-square test. ${ }^{\ddagger}$, data were presented as medians (inter-quartiles) and compared using the Mann-Whitney $U$ test. ACT, adjuvant chemotherapy; CEA, carcinoembryonic antigen; LI, labeling index; TNM, tumor-node-metastasis. 